\title{
Concurrent Chemoradiotherapy Versus Chemotherapy Alone for Unresectable Locally Advanced Pancreatic Cancer: A Retrospective Cohort Study
}

\section{Younak Choi, MD ${ }^{1, a}$ \\ Do-Youn Oh, MD, PhD ${ }^{1,2}$ \\ Kyubo Kim, MD, $\mathrm{PhD}^{3}$ \\ Eui Kyu Chie, MD, $P D^{3}$ \\ Tae-Yong Kim, MD, PhD ${ }^{1,2}$ \\ Kyung-Hun Lee, MD, $\mathrm{PhD}^{1,2}$ \\ Sae-Won Han, MD, PhD',2 \\ Seock-Ah Im, MD, PhD',2 \\ Tae-You Kim, MD, PhD',2 \\ Sung Whan $\mathrm{Ha}, \mathrm{MD}, \mathrm{PhD}^{3}$ \\ Yung-Jue Bang, MD, $P h D^{1,2}$}

\section{${ }^{1}$ Department of Internal Medicine,}

Seoul National University Hospital, Seoul,

${ }^{2}$ Cancer Research Institute,

Seoul National University

College of Medicine, Seoul,

${ }^{3}$ Department of Radiation Oncology,

Seoul National University Hospital, Seoul,

Korea

\author{
Correspondence: Do-Youn Oh, MD, PhD \\ Department of Internal Medicine, \\ Seoul National University Hospital, \\ 101 Daehak-ro, Jongno-gu, Seoul 03080, Korea \\ Tel: 82-2-2072-0701 \\ Fax: 82-2-762-9662 \\ E-mail: ohdoyoun@snu.ac.kr \\ Received June 24, 2015 \\ Accepted September 30, 2015 \\ Published Online October 16, 2015 \\ aPresent address: Department of Internal \\ Medicine, Dongguk University Gyeongju \\ Hospital, Gyeongju, Korea
}

\begin{abstract}
Purpose
The optimal treatment strategy for locally advanced pancreatic cancer (LAPC), particularly the role of concurrent chemoradiotherapy (CCRT), remains debatable. We compared the clinical outcomes of CCRT and palliative chemotherapy alone (CA) in patients with unresectable LAPC.
\end{abstract}

\section{Materials and Methods}

Patients with LAPC who were consecutively treated between 2003 and 2010 were included. Resectability was evaluated according to National Comprehensive Cancer Network ver. 1.2012. The clinical outcomes for each treatment group (CCRT vs. CA) were evaluated retrospectively.

\section{Results}

Sixty-three patients (58.9\%) and 44 patients (41.1\%) were treated with CCRT and CA, respectively. The CCRT cohort included patients who were treated with CCRT with or without chemotherapy backbone (CCRT alone, induction chemotherapy-CCRT, CCRT-maintenance chemotherapy, and induction-CCRT-maintenance chemotherapy). Median progression-free survival (PFS) and overall survival (OS) of all patients were 7.2 months and 13.1 months. PFS of the CCRT and CA groups was 9.0 months and 4.4 months, respectively $(p=0.020)$. OS of the CCRT and CA groups was 15.4 months and 9.3 months, respectively $(p=0.011)$. In multivariate analysis, the adjusted hazard ratio of CCRT was $0.536(p=0.003)$ for OS and $0.667(p=0.078)$ for PFS. Although the pattern of failure was similar in the CCRT and CA groups, the times to both local and distant failure were significantly longer in the CCRT group.

\section{Conclusion}

In patients with unresectable LAPC, those who underwent CCRT during their entire treatment courses had longer OS than patients treated with chemotherapy alone.

\section{Key words}

Pancreatic neoplasms, Chemoradiotherapy, Prognosis

\section{Introduction}

Pancreatic cancer (PC) is the fourth most common cause of cancer death in both Asian and western countries [1]. At the time of diagnosis, less than $20 \%$ of patients are eligible for curative surgery [2]. Among patients with initially inoperable PC, approximately $30 \%$ of patients present with locally advanced disease without distant metastasis [3]. PC has a dismal prognosis, with a 5-year overall survival (OS) rate of $5 \%$ [4].

The optimal treatment strategy for locally advanced pan- 
creatic cancer (LAPC) is still controversial, and the role of local control by concurrent chemoradiotherapy (CCRT) has not been established. Most clinical trials in the setting of palliative chemotherapy in PC have included both LAPC patients and metastatic $P C$ patients, altogether, and used the extent of disease as a stratification factor.

The superiority of CCRT to radiation alone was proven in 1981 [3]. Although the Gastrointestinal Tumor Study Group (GITSG) reported a higher efficacy of CCRT than chemotherapy alone (CA) in the 1980 's, the superiority of CCRT and CA continues to be debated $[5,6]$. After the introduction of gemcitabine for treatment of advanced $\mathrm{PC}$, treatment with gemcitabine alone resulted in longer survival than 5-fluorouracil (5-FU) based CCRT followed by maintenance gemcitabine (13 months vs. 8.6 months) in the FFCD-SFRO trial [7].

In contrast, in treatment with gemcitabine plus radiation longer survival was achieved compared to gemcitabine alone (11.1 months vs. 9.2 months) in the ECOG E4201 trial [8].

Induction chemotherapy prior to CCRT was introduced for achievement of both systemic and local control of possible unrecognized micro-metastases and to effectively identify the patients who might benefit from CCRT after induction chemotherapy $[9,10]$.

The introduction of new radiosensitizing agents with advanced radiotherapy techniques reduced toxicities; however, consensus regarding efficacy is still elusive [11,12]. Gemcitabine was reported to be a more potent radiosensitizer than 5-FU [13], and better outcomes were reported for capecitabine, another radiosensitizer, than gemcitabine [14]. However, those findings are insufficient to support a general consensus regarding the optimal treatment for LAPC.

Therefore, this study was conducted to evaluate the effects of adding CCRT to a chemotherapy backbone in LAPC.

\section{Materials and Methods}

\section{Study patients}

LAPC patients who were consecutively treated between 2003 and 2010 were included. Eligible patients had (1) histologically confirmed pancreatic adenocarcinoma, (2) no radiographic evidence of distant metastases, and (3) radiographic evidence of unresectability.

All computed tomography/magnetic resonance imaging image were reviewed for the unresectability, following the guidelines for LAPC of the National Comprehensive Cancer Network (NCCN) ver. 1.2012 [15]. Tumors met the criteria: tumors invaded or encased the aorta or encased the celiac axis or superior mesenteric artery by more than $180^{\circ}$ (abutted celiac axis at any degree in pancreatic head cancer) or demonstrated unreconstructable occlusion of the superior mesenteric vein or portal vein or extended to lymph nodes beyond the field of resection.

\section{Study methods}

\section{1) Treatment cohort}

Patients who underwent CA during the entire treatment course were included in the CA group. Patients who underwent CCRT during the entire treatment course were included in the CCRT group.

\section{2) Response evaluation}

Objective tumor response was evaluated according to the Response Evaluation Criteria In Solid Tumors (RECIST) ver. 1.1.

Body mass index (BMI) was calculated as body weight divided by height ${ }^{2}\left(\mathrm{~kg} / \mathrm{m}^{2}\right)$ measured the day of the first dose of chemotherapy. Tumors were staged according to the American Joint Committee on Cancer (AJCC) seventh edition.

\section{Statistical analysis}

OS, progression-free survival (PFS), best response, pattern of failure, and toxicities were evaluated according to treatment groups. OS was defined as the period from the start of treatment to death from any cause. PFS was defined as the period from the start of treatment to the progression of disease or death from any cause, with censoring of patients who are lost to follow-up.

The median OS and PFS were calculated using the KaplanMeier method. Between-group differences in demographic and clinical data were evaluated using Fisher exact test for categorical variables. The effect of multiple factors on survival, the hazard ratio (HR) and its $95 \%$ confidence interval (CI) were evaluated using Cox proportional hazards models. Each variable that was likely to be associated with survival, based on $\mathrm{p} \leq 0.1$ in the univariate analysis, was evaluated in the multivariate analysis. The survival of the two groups was compared using the log-rank test. All tests were 2-sided, and $\mathrm{p} \leq 0.05$ was considered statistically significant. Statistical analyses were performed using SPSS ver. 19.0 (IBM Co., Armonk, NY).

\section{Ethics}

This study was reviewed and approved by the Institu- 
Table 1. Clinical characteristics by treatment groups

\begin{tabular}{|c|c|c|c|c|}
\hline \multirow{2}{*}{ Characteristic } & \multicolumn{2}{|c|}{ CCRT vs. CA } & \multirow{2}{*}{ Total $(n=107)$} & \multirow{2}{*}{ p-value ${ }^{a}$} \\
\hline & CCRT $(n=63)$ & $\mathrm{CA}(\mathrm{n}=44)$ & & \\
\hline \multicolumn{5}{|l|}{ Sex } \\
\hline Male & $33(52.4)$ & $20(45.5)$ & $53(49.5)$ & 0.557 \\
\hline Female & $30(47.6)$ & $24(54.5)$ & $54(50.5)$ & \\
\hline \multicolumn{5}{|l|}{ Age (yr) } \\
\hline$\geq 60$ & $30(47.6)$ & $29(65.9)$ & $59(55.1)$ & 0.076 \\
\hline$<60$ & $33(52.4)$ & $15(34.1)$ & $48(44.9)$ & \\
\hline \multicolumn{5}{|l|}{ Performance } \\
\hline $0-1$ & $57(86.4)$ & $38(86.4)$ & $95(88.8)$ & 0.546 \\
\hline$\geq 2$ & $6(9.6)$ & $6(13.6)$ & $12(11.2)$ & \\
\hline \multicolumn{5}{|l|}{ T stage } \\
\hline 3 & $14(22.2)$ & 17 (38.6) & $31(29.0)$ & 0.084 \\
\hline 4 & $49(77.8)$ & $27(61.4)$ & $76(71.0)$ & \\
\hline \multicolumn{5}{|l|}{ N stage } \\
\hline 0 & $41(65.1)$ & $21(47.7)$ & $62(57.9)$ & 0.111 \\
\hline 1 & $22(34.9)$ & $23(52.3)$ & 45 (42.1) & \\
\hline \multicolumn{5}{|l|}{ Site } \\
\hline Head & $29(46.0)$ & $26(59.1)$ & $55(51.4)$ & 0.239 \\
\hline Corpse & $34(54.0)$ & $18(40.9)$ & $52(48.6)$ & \\
\hline \multicolumn{5}{|l|}{ Size (cm) } \\
\hline$\geq 3$ & $41(65.1)$ & $32(72.7)$ & $73(68.2)$ & 0.527 \\
\hline$<3$ & $22(34.9)$ & $12(27.3)$ & $34(31.8)$ & \\
\hline \multicolumn{5}{|c|}{ Initial BMI $\left(\mathrm{kg} / \mathrm{m}^{2}\right)$} \\
\hline$<20.0$ & $19(30.2)$ & $12(27.3)$ & $31(29.0)$ & 0.924 \\
\hline $20.0-24.9$ & $36(57.1)$ & $25(56.8)$ & $61(57.0)$ & \\
\hline$\geq 25.0$ & $8(12.7)$ & $7(15.9)$ & $15(14.0)$ & \\
\hline \multicolumn{5}{|l|}{ CA19-9 } \\
\hline Elevated & $46(73.0)$ & $37(84.1)$ & 83 (77.6) & 0.240 \\
\hline Normal & $17(27.0)$ & $7(15.9)$ & $24(22.4)$ & \\
\hline \multicolumn{5}{|l|}{ Albumin } \\
\hline Decreased & $11(17.5)$ & $10(22.7)$ & $21(19.6)$ & 0.622 \\
\hline Normal & $52(82.5)$ & $34(77.3)$ & $86(80.4)$ & \\
\hline \multicolumn{5}{|c|}{ No. of chemotherapy regimens } \\
\hline 1 & $31(49.2)$ & $29(65.9)$ & $60(56.1)$ & 0.114 \\
\hline$\geq 2$ & $32(50.8)$ & $15(34.1)$ & $47(43.9)$ & \\
\hline
\end{tabular}

Values are presented as number (\%). CCRT, concurrent chemoradiotherapy; CA, chemotherapy alone; BMI, body mass

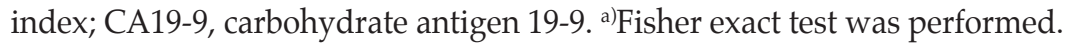

tional Review Board of Seoul National University Hospital (IRB No. H-1307-146-507). All studies were conducted according to ethical guidelines (Declaration of Helsinki) for biomedical research.

\section{Results}

\section{Patient characteristics}

A total of 107 patients with LAPC were identified retrospectively; their characteristics by treatment groups are shown in Table 1 . The median age was 60.7 years (range, 35.2 to 84.0 years) and most patients $(88.8 \%$ ) had a performance status of 0 or 1 . Thirty-one patients $(29.0 \%)$ had T3 lesions 


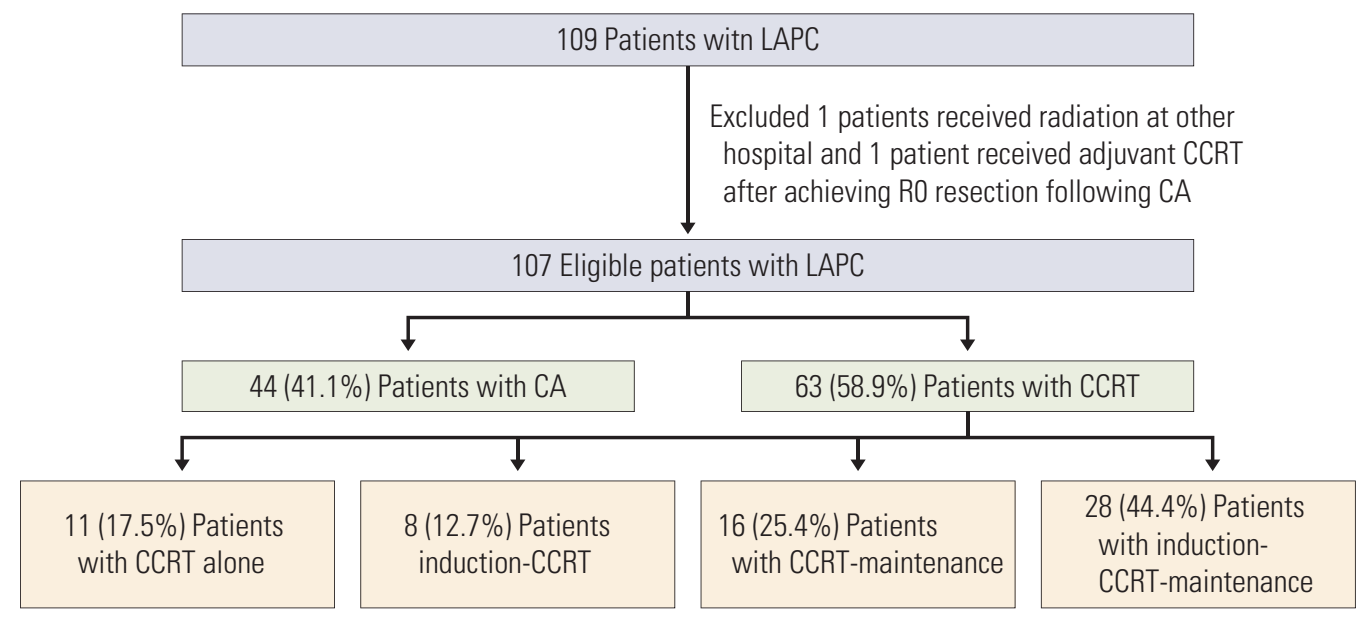

Fig. 1. Flow diagram for the study cohort. LAPC, locally advanced pancreatic cancer; CCRT, concurrent chemoradiotherapy, CA, chemotherapy alone.

and 76 patients $(71.0 \%)$ had T4 lesions, and 45 patients (42.1\%) had positive lymph nodes. Primary tumor sites were equally distributed in the head $(51.4 \%)$ and body $(48.6 \%)$ of the pancreas. Seventy-three patients $(68.2 \%)$ had primary tumors larger than $3 \mathrm{~cm}$. BMI distributions were $\mathrm{BMI}<20.0$ $(29.0 \%) ; 20.0 \leq \mathrm{BMI}<25.0(57.0 \%) ;$ and $\mathrm{BMI} \geq 25.0(14.0 \%)$.

\section{Treatment regimen}

Flow diagram for the study cohort is shown in Fig. 1 and treatments are summarized in Table 2. Sixty-three patients (58.9\%) underwent CCRT and 44 patients $(41.1 \%)$ underwent CA.

\section{1) CCRT cohort}

\section{(1) Induction chemotherapy}

Among the CCRT group, 36 patients $(57.1 \%)$ received a median of three cycles of induction chemotherapy (range, 1 to 9) prior to CCRT. The most commonly selected induction agents were gemcitabine-based doublets.

\section{(2) CCRT}

The prearranged total dose was $55.8 \mathrm{~Gy} / 31$ fractions delivered five times weekly, composed of $45 \mathrm{~Gy} / 25$ fractions delivered to the entire region and $10.8 \mathrm{~Gy} / 6$ fractions as a booster. Most patients $(n=56,88.9 \%)$ received a radiation dose $\geq 50$ Gy. Median total delivered radiation dose was 55.8 Gy (range, 32.4 to 59.4 Gy) delivered as 1.8 Gy per fraction (range, 1.6 to 2.0 Gy) over 6 weeks (median, 6.1 weeks; range 2.6 to 8.1 weeks). Radiosensitizers used during CCRT were gemcitabine $(n=17,27.0 \%), 5-\mathrm{FU}(\mathrm{n}=16,25.4 \%)$, and capecitabine $(n=30,47.6 \%)$.

\section{(3) Maintenance chemotherapy}

Patients without progression after CCRT received additional maintenance chemotherapy $(\mathrm{n}=44,69.8 \%)$ using a median of three cycles (range, 1 to 17 cycles). The most frequently used maintenance chemotherapy agents were also gemcitabine-based regimens $(\mathrm{n}=38,86.3 \%)$.

\section{2) CA cohort}

Among the patients in the CA group, gemcitabine-based regimen was used in $97.7 \%$ of patients. Median chemotherapy cycle and dose intensity were three cycles (range, 1 to 18 cycles) and $100 \%$ (range, $60 \%$ to $100 \%$ ), respectively.

The baseline characteristics showed a similar distribution in the CCRT and CA groups (Table 1).

\section{Treatment outcomes}

The median duration of follow-up for all patients was 12.6 months (range, 2.0 to 58.3 months), and the median OS and PFS were 13.1 months (95\% CI, 11.6 to 14.6 months) and 7.2 months (95\% CI, 6.2 to 8.3 months), respectively. Two patients who underwent CCRT after first-line failure were excluded from the analysis of PFS for first-line treatment. 
Table 2. Details of treatment regimens

$\begin{array}{ll}\text { Characteristic } & \text { No. of patients }(\%)\end{array}$

CCRT group $(\mathrm{n}=63)$

Induction chemotherapy $(\mathrm{n}=63)$

Administered

$36(57.1)$

Not administered

$27(42.9)$

DI of induction chemotherapy $(\%)(\mathrm{n}=36)$

100

$18(50.0)$

$<100$

$18(50.0)$

Agents of induction chemotherapy $(n=36)$

Gemcitabine/ cisplatin

$31(86.1)$

Gemcitabine/capecitabine

$1(2.8)$

Gemcitabine/ oxaliplatin

$1(2.8)$

Gemcitabine alone

Radiosensitizer ( $\mathrm{n}=63$ )

Gemcitabine

$17(27.0)$

5-FU

$16(25.4)$

Capecitabine

$30(47.6)$

Total radiation dose (Gy) ( $\mathrm{n}=63$ )

$<50$

7 (11.1)

50-60

$56(88.9)$

Radiation dose per fraction (Gy) ( $\mathrm{n}=63)$

1.6

$1(1.6)$

$1.8 \quad 52(82.5)$

2

$10(15.9)$

Maintenance chemotherapy $(\mathrm{n}=63)$

Administered

$44(69.8)$

Not administered

$19(30.2)$

DI of maintenance chemotherapy $(\%)(n=44)$

100

$<100$ (70-87.5)

$21(47.7)$

Agents of maintenance chemotherapy $(\mathrm{n}=44)$

Gemcitabine/ cisplatin

$9(20.4)$

Gemcitabine/capecitabine

$3(6.8)$

Gemcitabine/5-FU

$1(2.3)$

Gemcitabine alone

$25(56.8)$

5-FU/leucovorin

$4(9.1)$

5-FU/cisplatin

$1(2.3)$

Capecitabine alone

1 (2.3)

CA group $(n=44)$

DI of chemotherapy $(\%)(n=44)$

100

$28(63.6)$

$<100$ (70-87.5)

$16(36.4)$

Agents of chemotherapy $(n=44)$

Gemcitabine/cisplatin

$18(40.9)$

Gemcitabine/capecitabine

$10(22.7)$

Gemcitabine/oxaliplatin

$4(9.1)$

Gemcitabine/erlotinib

2 (4.5)

Gemcitabine/capecitabine/erlotinib

$1(2.3)$

Gemcitabine/S-1

1 (2.3)

Gemcitabine alone

7 (15.9)

S-1

$1(2.3)$

CCRT, concurrent chemoradiotherapy; 5-FU, 5-fluorouracil; DI, dose intensity; CA, chemotherapy alone. 


\section{Outcomes analysis by treatment group}

First-line treatment outcomes of CCRT and CA groups are summarized in Table 3.

\section{1) Response}

In the CCRT group, three patients $(4.9 \%)$ had complete response (CR), 18 (29.5\%) had partial response (PR), and 37 $(60.7 \%)$ had stable disease (SD). In the CA group, one patient (2.3\%) had CR, seven (15.9\%) had PR, and 18 (40.9\%) had SD.

\section{2) OS}

The median OS was 15.4 months (95\% CI, 13.2 to 17.6 months) in the CCRT group and 9.3 months (95\% CI, 6.6 to 12.0 months) in the CA group ( $\mathrm{p}=0.011$ ) (Table 4, Fig. 2A). The 1-year OS was $69.1 \%$ in the CCRT group and $37.9 \%$ in the CA group. The median OS of patients who underwent CCRT alone, induction-CCRT, CCRT-maintenance, and induction-CCRT-maintenance was 10.9 months (95\% CI, 6.4 to 15.4 months), 9.2 months (95\% CI, 1.7 to 16.8 months), 17.0 months (95\% CI, 14.3 to 19.8 months), and 17.0 months (95\% CI, 15.1 to 18.9 months), respectively ( $p=0.010$ ). CCRT with induction chemotherapy did not differ significantly from CCRT without induction chemotherapy in OS (16.4 months vs. 13.6 months; HR, 0.840 ; $95 \%$ CI, 0.492 to $1.435 ; \mathrm{p}=0.524)$. However, CCRT with maintenance chemotherapy resulted in significantly longer OS than CCRT without maintenance chemotherapy (17.0 months vs. 9.2 months; HR, 0.449; $95 \%$ CI, 0.251 to $0.801 ; \mathrm{p}=0.007$ ).

\section{3) PFS}

The median PFS was 9.0 months (95\% CI, 7.0 to 11.0 months) in the CCRT group and 4.4 months $(95 \%$ CI, 0.9 to 8.0 months) in the CA group ( $\mathrm{p}=0.020)$. Six-month PFS was $68.3 \%$ and $47.4 \%$ and 1 -year PFS was $28.6 \%$ and $13.1 \%$, respectively (Table 4, Fig. 2B). The PFS of patients who underwent CCRT alone, induction-CCRT, CCRT-maintenance, and induction-CCRT-maintenance was 3.0 months (95\% CI, 2.3 to 3.7 months), 5.3 months (95\% CI, 4.5 to 6.1 months), 6.9 months (95\% CI, 2.4 to 11.5 months), and 11.4 months (95\% CI, 10.2 to 12.7 months), respectively $(\mathrm{p}<0.001)$. The use of induction and maintenance chemotherapy prolonged PFS (10.8 months vs. 4.7 months; HR, $0.449 ; 95 \% \mathrm{CI}, 0.261$ to $0.773 ; \mathrm{p}=0.004)$ and (10.8 months vs. 4.5 months; HR, 0.322; 95\% CI, 0.177 to $0.585 ; \mathrm{p}<0.001$ ), respectively. Only one patient in the CCRT group converted to resectability, underwent margin-negative resection after CCRT, and had an OS of 17.0 months and PFS of 6.9 months.
Table 3. Response to first-line treatment

\begin{tabular}{|c|c|}
\hline Characteristic & No. of patients $(\%)$ \\
\hline \multicolumn{2}{|l|}{ CCRT group $(n=61)$} \\
\hline \multicolumn{2}{|l|}{ Treatment scheme $(\mathrm{n}=61)$} \\
\hline CCRT alone & $10(16.4)$ \\
\hline Induction-CCRT & $8(13.1)$ \\
\hline CCRT-maintenance & $15(24.6)$ \\
\hline Induction-CCRT-maintenance & $28(45.9)$ \\
\hline \multicolumn{2}{|c|}{ BR to induction chemotherapy $(n=36)$} \\
\hline PR & $5(13.9)$ \\
\hline SD & $31(86.1)$ \\
\hline \multicolumn{2}{|l|}{ BR to CCRT $(n=61)$} \\
\hline $\mathrm{CR}$ & $1(1.6)$ \\
\hline PR & $15(24.6)$ \\
\hline SD & $39(64.0)$ \\
\hline PD & $6(9.8)$ \\
\hline \multicolumn{2}{|l|}{ BR to entire CCRT regimen $(n=61)$} \\
\hline $\mathrm{CR}$ & $3(4.9)$ \\
\hline PR & $18(29.5)$ \\
\hline SD & $37(60.7)$ \\
\hline PD & $3(4.9)$ \\
\hline \multicolumn{2}{|l|}{ Pattern of failure $(n=59)$} \\
\hline Local & $21(35.6)$ \\
\hline Systemic & $23(39.0)$ \\
\hline Both & 7 (11.9) \\
\hline NA & $8(13.5)$ \\
\hline \multicolumn{2}{|l|}{ CA group $(n=44)$} \\
\hline \multicolumn{2}{|l|}{ BR to first-line treatment $(n=44)$} \\
\hline CR & $1(2.3)$ \\
\hline PR & $7(15.9)$ \\
\hline SD & $18(40.9)$ \\
\hline PD & $12(27.3)$ \\
\hline NA & $6(13.6)$ \\
\hline \multicolumn{2}{|l|}{ Pattern of failure $(n=43)$} \\
\hline Local & $12(27.3)$ \\
\hline Systemic & $13(29.5)$ \\
\hline Both & $4(9.1)$ \\
\hline NA & $14(34.1)$ \\
\hline
\end{tabular}

CCRT, concurrent chemoradiotherapy; $\mathrm{BR}$, best response; $\mathrm{PR}$, partial response; $\mathrm{SD}$, stable disease; $\mathrm{CR}$, complete response; $\mathrm{PD}$, progressive disease; $\mathrm{NA}$, not assessable; $\mathrm{CA}$, chemotherapy alone.

\section{4) Patterns of failure}

The pattern of progression (local failure and/or distant metastasis) is shown in Table 4. Among assessable patients, the frequency of local failure, with or without distant progression, did not differ; $54.9 \%$ and $58.6 \%$ in the CCRT and 
Table 4. Summary of response and survival by treatment groups

\begin{tabular}{|c|c|c|c|}
\hline \multirow{2}{*}{ Characteristic } & \multicolumn{2}{|c|}{ CCRT vs. CA (n=105) } & \multirow{2}{*}{ p-value } \\
\hline & CCRT $(n=61)$ & $\mathrm{CA}(\mathrm{n}=44)$ & \\
\hline First-line treatment & 61 & 44 & \\
\hline \multicolumn{4}{|l|}{ Best response } \\
\hline ORR & $21(34.4)$ & $8(18.2)$ & $0.079^{\mathrm{a})}$ \\
\hline DCR & $58(95.1)$ & $26(59.1)$ & $<0.001^{\text {a) }}$ \\
\hline \multicolumn{4}{|l|}{ Pattern of PD (of accessible patients) ( $\mathrm{n}=82$ ) } \\
\hline Local & $28(54.9)$ & $17(58.6)$ & $0.817^{\mathrm{a})}$ \\
\hline Systemic & $30(58.8)$ & $17(58.6)$ & $1.000^{\mathrm{a})}$ \\
\hline \multicolumn{4}{|l|}{ Median survival (mo) } \\
\hline First-line PFS (95\% CI) & $9.0(7.0-11.0)$ & $4.4(0.9-8.0)$ & $0.020^{\mathrm{b})}$ \\
\hline Time to local failure $(95 \% \mathrm{CI})(\mathrm{n}=46)$ & $10.5(8.5-12.4)$ & $3.7(1.4-5.9)$ & $0.001^{\mathrm{b})}$ \\
\hline Time to distant failure $(95 \% \mathrm{CI})(\mathrm{n}=48)$ & $7.8(5.1-10.5)$ & $5.0(1.6-8.4)$ & $0.001^{\mathrm{b})}$ \\
\hline OS $(95 \%$ CI $)(\mathrm{n}=107)$ & $15.4(13.2-17.6)$ & $9.3(6.6-12.0)$ & $0.011^{\mathrm{b})}$ \\
\hline
\end{tabular}

Values are presented as number (\%). CCRT, concurrent chemoradiotherapy; CA, chemotherapy alone; ORR, objective response rate; DCR, disease control rate; PD, progression; PFS, progression-free survival; CI, confidential interval; OS, overall survival. ${ }^{a)}$ Fisher exact test was performed, ${ }^{b}$ Log-rank test was performed.

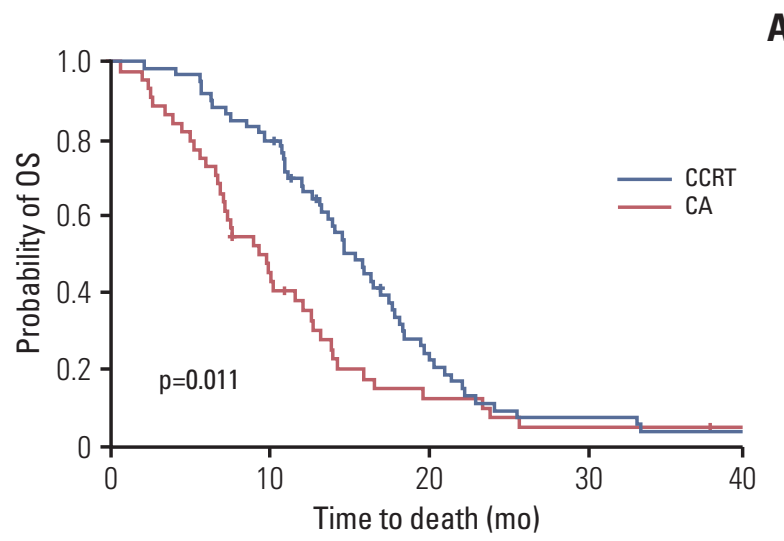

A

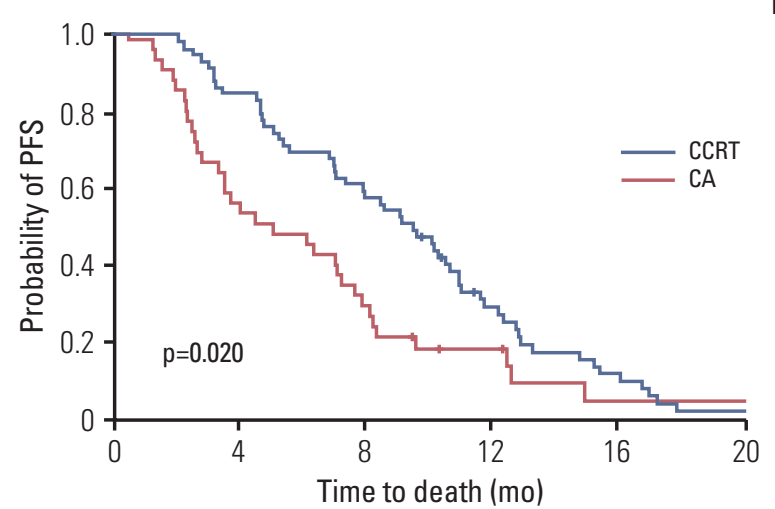

Fig. 2. Overall survival (OS) and progression-free survival (PFS) by first-line treatment modality. (A) Median OS was 15.4 months (95\% confidence interval [CI], 13.2 to 17.6 months) in the concurrent chemoradiotherapy (CCRT) group and 9.3 months (95\% CI, 6.6 to 12.0 months) in the chemotherapy alone (CA) group ( $\mathrm{p}=0.011)$. (B) Median PFS was 9.0 months (95\% CI, 7.0 to 11.0 months) in the CCRT group and 4.0 months (95\% CI, 1.6 to 6.3 months) in the CA group ( $p=0.010$ ).

CA groups, respectively ( $\mathrm{p}=0.817)$. However, median time to local progression was longer, by 6.8 months, in the CCRT group than in the CA group $(p<0.001)$. The frequency of systemic failure, with or without local progression, was also similar, $58.8 \%$ in the CCRT group and $58.6 \%$ in the CA group $(p=1.000)$. Median time to distant progression was also longer, by 2.8 months, in the CCRT group than in the CA group ( $\mathrm{p}=0.001$ ) (Table 4). However, in comparison of the CCRT alone and CA groups, no significant difference was found in survival and pattern of failure (OS: HR, 0.880; $\mathrm{p}=0.718$; time to local failure: $H R, 1.255 ; \mathrm{p}=0.665$; time to systemic failure: HR, 0.749; $\mathrm{p}=0.654)$. In all patients, the most frequent sites of distant failure were liver $(30.9 \%)$, peritoneum $(27.2 \%)$, and lung $(12.3 \%)$. 
Table 5. Analysis of potential prognostic factors for overall survival

\begin{tabular}{|c|c|c|c|c|c|c|c|}
\hline \multirow{2}{*}{ Clinical factor } & \multirow{2}{*}{ mOS (mo) } & \multicolumn{3}{|c|}{ Univariate analysis } & \multicolumn{3}{|c|}{ Multivariate analysis } \\
\hline & & HR & $95 \% \mathrm{CI}$ & p-value ${ }^{a)}$ & HR & $95 \% \mathrm{CI}$ & p-value ${ }^{\text {b) }}$ \\
\hline \multicolumn{8}{|l|}{ Age (yr) } \\
\hline$\geq 60$ & - & 1.135 & $0.759-1.698$ & 0.536 & - & - & - \\
\hline$<60$ & - & 1 & & & - & & \\
\hline \multicolumn{8}{|l|}{ Performance } \\
\hline$\geq 2$ & - & 1.434 & $0.760-2.707$ & 0.266 & - & - & - \\
\hline $0-1$ & - & 1 & & & - & & \\
\hline \multicolumn{8}{|l|}{ T stage } \\
\hline 4 & - & 0.961 & $0.616-1.499$ & 0.861 & - & - & - \\
\hline 3 & - & 1 & & & - & & \\
\hline \multicolumn{8}{|l|}{ N stage } \\
\hline 1 & - & 1.165 & $0.776-1.749$ & 0.462 & - & - & - \\
\hline 0 & - & 1 & & & - & & \\
\hline \multicolumn{8}{|l|}{ Site } \\
\hline Corpse & - & 0.816 & $0.547-1.217$ & 0.319 & - & - & - \\
\hline Head & - & 1 & & & - & & \\
\hline \multicolumn{8}{|l|}{ Size $(\mathrm{cm})$} \\
\hline$\geq 3$ & - & 1.302 & $0.841-2.014$ & 0.236 & - & - & - \\
\hline$<3$ & - & 1 & & & - & & \\
\hline Initial BMI $\left(\mathrm{kg} / \mathrm{m}^{2}\right)$ & & & & 0.021 & & & 0.097 \\
\hline$<20.0$ & 10.7 & 1 & & Reference & 1 & & Reference \\
\hline $20.0-24.9$ & 14.2 & 0.532 & $0.337-0.840$ & 0.007 & 0.601 & 0.374-0.965 & 0.035 \\
\hline$\geq 25.0$ & 12.1 & 0.543 & $0.277-1.067$ & 0.077 & 0.781 & $0.374-1.631$ & 0.511 \\
\hline \multicolumn{8}{|l|}{ CCRT } \\
\hline Yes & 15.4 & 0.589 & $0.392-0.885$ & 0.011 & 0.536 & $0.355-0.809$ & 0.003 \\
\hline No & 9.3 & 1 & & & 1 & & \\
\hline \multicolumn{8}{|l|}{ CA19-9 } \\
\hline Elevated & 11.5 & 1.979 & $1.201-3.261$ & 0.007 & 1.877 & $1.077-3.272$ & 0.026 \\
\hline Normal & 17 & 1 & & & 1 & & \\
\hline \multicolumn{8}{|l|}{ Albumin } \\
\hline Decreased & 6.3 & 3.033 & $1.807-5.089$ & $<0.001$ & 3.148 & $1.863-5.320$ & $<0.001$ \\
\hline Normal & 13.9 & 1 & & & 1 & & \\
\hline
\end{tabular}

mOS, median overall survival; $\mathrm{HR}$, hazard ratio; $\mathrm{CI}$, confidential interval; $\mathrm{BMI}$, body mass index; CCRT, concurrent chemoradiotherapy; CA19-9, carbohydrate antigen 19-9. ${ }^{\text {a) }}$ Cox proportional hazard model was performed, b) ${ }^{\text {box }}$ proportional hazard model adjusted for BMI, CCRT, CA 19-9, and albumin was performed.

\section{Analysis of prognostic factors}

Table 5 shows the results of the analysis of possible prognostic factors for OS. In the univariate analysis, BMI $(\mathrm{p}=0.021)$, CCRT $(\mathrm{p}=0.011)$, carbohydrate antigen 19-9 (CA19-9) $(\mathrm{p}=0.007)$, and albumin $(\mathrm{p}<0.001)$ showed significant association with OS. In the multivariate analysis, elevated CA19-9 (HR, 1.877; 95\% CI, 1.077 to 3.272; $\mathrm{p}=0.026$ ) and decreased albumin levels (HR, 3.148; 95\% CI, 1.863 to 5.320; $\mathrm{p}<0.001)$ conferred a higher risk for death, whereas CCRT (HR, $0.536 ; 95 \% \mathrm{CI}, 0.355$ to $0.809 ; \mathrm{p}=0.003$ ) significantly lowered the risk.
In the univariate analysis, the significant prognostic factors for PFS were T stage $(\mathrm{p}=0.001)$, N stage $(\mathrm{p}=0.021)$, initial BMI $(\mathrm{p}=0.030)$, and CCRT $(\mathrm{p}=0.020)$. In the multivariate analysis, T4 (HR, 0.504; 95\% CI, 0.308 to 0.825; $\mathrm{p}=0.006$ ), positive lymph node (HR, 1.664; 95\% CI, 1.049 to 2.639; $\mathrm{p}=0.030$ ), and normal range of initial BMI (HR, 0.590; 95\% CI, 0.350 to $0.995 ; \mathrm{p}=0.048$ ) showed association with PFS. CCRT (HR, $0.667 ; 95 \% \mathrm{CI}, 0.426$ to $1.046 ; \mathrm{p}=0.078$ ) and decreased albumin (HR, 1.662; 95\% CI, 0.931 to $2.969 ; \mathrm{p}=0.086)$ tended to showed association with longer and shorter PFS, respectively (Table 6). 
Table 6. Analysis of potential prognostic factors for progression-free survival

\begin{tabular}{|c|c|c|c|c|c|c|c|}
\hline \multirow{2}{*}{ Clinical factor } & \multirow{2}{*}{ mOS (mo) } & \multicolumn{3}{|c|}{ Univariate analysis } & \multicolumn{3}{|c|}{ Multivariate analysis } \\
\hline & & HR & $95 \% \mathrm{CI}$ & p-value ${ }^{a)}$ & HR & $95 \% \mathrm{CI}$ & p-value ${ }^{\text {b) }}$ \\
\hline \multicolumn{8}{|l|}{ Age (yr) } \\
\hline$\geq 60$ & 6.2 & 1.127 & $0.740-1.716$ & 0.576 & - & - & - \\
\hline$<60$ & 8.3 & 1 & & & - & & \\
\hline \multicolumn{8}{|l|}{ Performance } \\
\hline$\geq 2$ & 6 & 0.969 & $0.4514-1.826$ & 0.922 & - & - & - \\
\hline $0-1$ & 7.7 & 1 & & & - & & \\
\hline \multicolumn{8}{|l|}{ T stage } \\
\hline 4 & 8.4 & 0.432 & $0.269-0.694$ & 0.001 & 0.504 & $0.308-0.825$ & 0.006 \\
\hline 3 & 4.4 & 1 & & & 1 & & \\
\hline \multicolumn{8}{|l|}{ N stage } \\
\hline 1 & 5.2 & 1.641 & $1.076-2.502$ & 0.021 & 1.664 & $1.049-2.639$ & 0.03 \\
\hline 0 & 8.3 & 1 & & & 1 & & \\
\hline \multicolumn{8}{|l|}{ Site } \\
\hline Corpse & 8.0 & 0.860 & $0.568-1.304$ & 0.478 & - & - & - \\
\hline Head & 6.7 & 1 & & & - & & \\
\hline \multicolumn{8}{|l|}{ Size $(\mathrm{cm})$} \\
\hline$\geq 3$ & 7.1 & 0.969 & $0.622-1.509$ & 0.889 & - & - & - \\
\hline$<3$ & 7.7 & 1 & & & - & & \\
\hline Initial BMI $\left(\mathrm{kg} / \mathrm{m}^{2}\right)$ & & & & 0.030 & & & 0.116 \\
\hline$<20.0$ & 5.0 & 1 & & Reference & 1 & & Reference \\
\hline $20.0-24.9$ & 8.3 & 0.527 & $0.323-0.860$ & 0.010 & 0.590 & $0.350-0.995$ & 0.048 \\
\hline$\geq 25.0$ & 7.8 & 0.510 & $0.250-1.039$ & 0.064 & 0.822 & $0.379-1.780$ & 0.618 \\
\hline \multicolumn{8}{|l|}{ CCRT } \\
\hline Yes & 9.0 & 0.598 & $0.389-0.922$ & 0.020 & 0.667 & $0.426-1.046$ & 0.078 \\
\hline No & 4.4 & 1 & & & 1 & & \\
\hline \multicolumn{8}{|l|}{ CA19-9 } \\
\hline Elevated & 6.9 & 1.543 & $0.944-2.521$ & 0.084 & 1.295 & $0.754-2.224$ & 0.349 \\
\hline Normal & 10.5 & 1 & & & 1 & & \\
\hline \multicolumn{8}{|l|}{ Albumin } \\
\hline Decreased & 3.2 & 1.658 & $0.973-2.826$ & 0.063 & 1.662 & $0.931-2.969$ & 0.086 \\
\hline Normal & 8.0 & 1 & & & 1 & & \\
\hline
\end{tabular}

mPFS, median progression free survival; $\mathrm{HR}$, hazard ratio; $\mathrm{CI}$, confidential interval; $\mathrm{BMI}$, body mass index; CCRT, concurrent

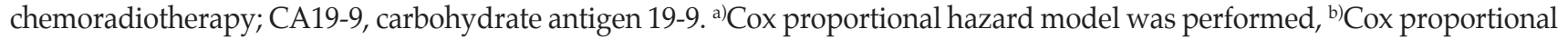
hazard model adjusted for T stage, N stage, BMI, CCRT, CA 19-9, and albumin was performed.

\section{Discussion}

The question of whether CCRT or CA is superior for LAPC has been debated for decades. In this study, we demonstrated an advantage of CCRT. Using multivariate analysis, we found that addition of CCRT in the chemotherapy backbone had excellent prognostic implications for treatment of LAPC patients. Further examination showed that better outcomes could be achieved by sequential treatment of CCRTmaintenance chemotherapy.

In addition, although the CCRT and CA groups showed similar patterns of failure, time to distant progression and to time to local progression were also significantly longer in the CCRT group.

Previous clinical trials focusing on combined radiosensitizers have reported conflicting results. Although gemcitabine was reported to be a more potent radiosensitizer than 5 -FU [13], these results were inconsistent [11,16]. In the SCALOP trial, the use of capecitabine, a radiosensitizer, resulted in a better outcome than gemcitabine [14]. However, further controlled trials comparing the efficacy of radiosensitizers are needed.

For more than 30 years, many studies have investigated 
the optimal treatment of LAPC. Some studies supported the superiority of CCRT over CA [5,8,9], but others did not agree with CCRT's superiority $[7,17]$. In this study, longer OS was observed with CCRT than with CA and longer PFS by use of an induction and/or maintenance chemotherapy backbone in the CCRT regimen. Of note, the median PFS of patients treated with CCRT alone was only 3.0 months. In previous studies of LAPC, the use of CCRT after disease control with induction chemotherapy prolonged both OS and PFS $[9,18]$. These findings support the hypothesis that combined chemotherapy can suppress distant metastases caused by early micro-metastases [19]. Based on analysis of a subgroup of patients of the LAP07 trial, a recent report showed that detection of circulating tumor cells (CTCs) for evaluation of micrometastatic disease was a promising prognostic tool. CTC positivity at baseline and / or at 2 months was associated with a shorter survival $(\mathrm{p}=0.01)$ [10].

Among the treatment modalities for PC, resection with a negative margin provides the most favorable prognosis. Because some studies reported their experience with R0 resection in LAPC patients, after pre-operative CCRT allowed down-staging, interest in the neoadjuvant role of CCRT for patients with LAPC and borderline resectable pancreatic cancer (BRPC) patient is increasing [20].

We observed that CCRT, when added to a combined chemotherapy backbone, is clearly beneficial for LAPC patients. We demonstrated that maintenance chemotherapy lengthened both OS and PFS, but we could not prove the efficacy of induction chemotherapy.
The strengths of this study were (1) only initially unresectable LAPC patients were included. BRPC patients with a higher possibility of $\mathrm{R} 0$ resection and better prognosis were excluded. (2) We avoided possible confounding by FOLFIRINOX, which is the most powerful first-line treatment regimen and has no credible efficacy data as second-line treatment. The limitations of this trial originated from the retrospective design. We could not obtain reliable data on toxicities.

\section{Conclusion}

In conclusion, our study strongly suggests that strengthening the early treatment by addition of CCRT in the chemotherapy backbone during treatment of LAPC patients conferred better treatment outcomes. Further controlled trials are warranted to evaluate the role of CCRT and the efficacy of combined agents.

\section{Conflicts of Interest}

Conflict of interest relevant to this article was not reported.

\section{References}

1. Kris MG, Benowitz SI, Adams S, Diller L, Ganz P, Kahlenberg MS, et al. Clinical cancer advances 2010: annual report on progress against cancer from the American Society of Clinical Oncology. J Clin Oncol. 2010;28:5327-47.

2. Mayahara H, Ito Y, Morizane C, Ueno H, Okusaka T, Kondo $S$, et al. Salvage chemoradiotherapy after primary chemotherapy for locally advanced pancreatic cancer: a single-institution retrospective analysis. BMC Cancer. 2012;12:609.

3. Moertel CG, Frytak S, Hahn RG, O'Connell MJ, Reitemeier RJ, Rubin J, et al. Therapy of locally unresectable pancreatic carcinoma: a randomized comparison of high dose (6000 rads) radiation alone, moderate dose radiation (4000 rads + 5-fluorou-racil), and high dose radiation + 5-fluorouracil: The Gastrointestinal Tumor Study Group. Cancer. 1981;48:1705-10.

4. Hariharan D, Saied A, Kocher HM. Analysis of mortality rates for pancreatic cancer across the world. HPB (Oxford). 2008; 10:58-62.

5. Treatment of locally unresectable carcinoma of the pancreas: comparison of combined-modality therapy (chemotherapy plus radiotherapy) to chemotherapy alone. Gastrointestinal Tumor Study Group. J Natl Cancer Inst. 1988;80:751-5.

6. Cohen SJ, Dobelbower R Jr, Lipsitz S, Catalano PJ, Sischy B, Smith TJ, et al. A randomized phase III study of radiotherapy alone or with 5-fluorouracil and mitomycin- $C$ in patients with locally advanced adenocarcinoma of the pancreas: Eastern Cooperative Oncology Group study E8282. Int J Radiat Oncol Biol Phys. 2005;62:1345-50.

7. Chauffert B, Mornex F, Bonnetain F, Rougier P, Mariette C, Bouche $\mathrm{O}$, et al. Phase III trial comparing intensive induction chemoradiotherapy (60 Gy, infusional 5-FU and intermittent cisplatin) followed by maintenance gemcitabine with gemcitabine alone for locally advanced unresectable pancreatic cancer. Definitive results of the 2000-01 FFCD/SFRO study. Ann Oncol. 2008;19:1592-9.

8. Loehrer PJ Sr, Feng Y, Cardenes H, Wagner L, Brell JM, Cella $\mathrm{D}$, et al. Gemcitabine alone versus gemcitabine plus radiother- 
apy in patients with locally advanced pancreatic cancer: an Eastern Cooperative Oncology Group trial. J Clin Oncol. 2011; 29:4105-12.

9. Huguet F, Andre T, Hammel P, Artru P, Balosso J, Selle F, et al. Impact of chemoradiotherapy after disease control with chemotherapy in locally advanced pancreatic adenocarcinoma in GERCOR phase II and III studies. J Clin Oncol. 2007;25: 326-31.

10. Bidard FC, Huguet F, Louvet C, Mineur L, Bouche O, Chibaudel B, et al. Circulating tumor cells in locally advanced pancreatic adenocarcinoma: the ancillary CirCe 07 study to the LAP 07 trial. Ann Oncol. 2013;24:2057-61.

11. Wilkowski R, Boeck S, Ostermaier S, Sauer R, Herbst M, Fietkau $\mathrm{R}$, et al. Chemoradiotherapy with concurrent gemcitabine and cisplatin with or without sequential chemotherapy with gemcitabine/cisplatin vs chemoradiotherapy with concurrent 5-fluorouracil in patients with locally advanced pancreatic cancer-a multi-centre randomised phase II study. Br J Cancer. 2009;101:1853-9.

12. Zhu CP, Shi J, Chen YX, Xie WF, Lin Y. Gemcitabine in the chemoradiotherapy for locally advanced pancreatic cancer: a meta-analysis. Radiother Oncol. 2011;99:108-13.

13. Li CP, Chao Y, Chi KH, Chan WK, Teng HC, Lee RC, et al. Concurrent chemoradiotherapy treatment of locally advanced pancreatic cancer: gemcitabine versus 5-fluorouracil, a randomized controlled study. Int J Radiat Oncol Biol Phys. 2003;57:98-104.

14. Mukherjee S, Hurt CN, Bridgewater J, Falk S, Cummins S, Wasan $\mathrm{H}$, et al. Gemcitabine-based or capecitabine-based chemoradiotherapy for locally advanced pancreatic cancer
(SCALOP): a multicentre, randomised, phase 2 trial. Lancet Oncol. 2013;14:317-26.

15. National Comprehensive Cancer Network. NCCN guidelines for treatment of cancer by site. Fort Washington, PA: National Comprehensive Cancer Network; 2014.

16. Crane CH, Abbruzzese JL, Evans DB, Wolff RA, Ballo MT, Delclos $\mathrm{M}$, et al. Is the therapeutic index better with gemcitabine-based chemoradiation than with 5-fluorouracil-based chemoradiation in locally advanced pancreatic cancer? Int J Radiat Oncol Biol Phys. 2002;52:1293-302.

17. Klaassen DJ, MacIntyre JM, Catton GE, Engstrom PF, Moertel CG. Treatment of locally unresectable cancer of the stomach and pancreas: a randomized comparison of 5-fluorouracil alone with radiation plus concurrent and maintenance 5-fluorouracil: an Eastern Cooperative Oncology Group study. J Clin Oncol. 1985;3:373-8.

18. Krishnan S, Rana V, Janjan NA, Varadhachary GR, Abbruzzese JL, Das P, et al. Induction chemotherapy selects patients with locally advanced, unresectable pancreatic cancer for optimal benefit from consolidative chemoradiation therapy. Cancer. 2007;110:47-55.

19. Ajani JA, Walsh G, Komaki R, Morris J, Swisher SG, Putnam JB Jr, et al. Preoperative induction of CPT-11 and cisplatin chemotherapy followed by chemoradiotherapy in patients with locoregional carcinoma of the esophagus or gastroesophageal junction. Cancer. 2004;100:2347-54.

20. Lloyd S, Chang BW. A comparison of three treatment strategies for locally advanced and borderline resectable pancreatic cancer. J Gastrointest Oncol. 2013;4:123-30. 